\title{
SAGE Thoughts on Aging
}

\author{
Thomas E. Johnson \\ Institute for Behavioral Genetics, University of Colorado at Boulder, Boulder, Colorado 80309, USA
}

he scientific method, and genetic analysis in particular, is based upon identifying variations between individuals of the same species. The study of Jones et al. in this issue reveals variation in transcript abundance between two developmental stages of the nematode Caenorhabditis elegans. In this case, the variation is not genetically specified but is induced by the environment as part of a shift to an alternate developmental form, the dauer larva. In this type of whole-genome analyses, it is assumed that such studies would reveal differences in transcript abundance that would be causally associated with distinct molecular and morphological transformations driving development. Much of this paper is conjecture about how the observed differences in transcript abundance specify observed differences in longevity (or, more precisely, in mortality rate).

C. elegans owes its existence as a species (a very successful, cosmopolitan species at that) to its ability to perform two critical tasks: finding new food resources and producing new progeny. In the wild, these events are temporally separated and involve continual transitions between the migratory foodseeking stage (the dauer) and the reproductive stage most commonly studied in the lab. The long-lived age-1mutant (see Braeckman et al. [2001] for the most recent comprehensive review on long-lived mutants), has a subtle change in its ability to transit between the dauer and the reproductive stages. This alteration does not affect its ability to grow when on plentiful food; however, under varying food conditions the age- 1 mutation leads to a rapid loss of the mutant form in competition with wild type (Walker et al. 2000).

The dauer is not an immobile spore; it is highly migratory and this stage is almost certainly responsible for the cosmopolitan nature of this 1-mm invertebrate. Dauers survive for several months, showing negligible senescence (T.E. Johnson and P.M. Tedesco, unpubl.), and those that survive have normal lifespans and ability to reproduce if returned to a food source (Klass and Hirsh 1976). To

\section{E-MAIL johnsont@colorado.edu; FAX (303)} 4928063.

Article and publication are at http://www.genome. org/cgi/doi/10.1101/gr.201101. accomplish this feat, the dauer dries itself out, puts on an extra cuticle, changes its normal behavior from that of negative to positive geotaxis, and synthesizes a variety of proteins thought to protect the dauer in its attempt to survive long enough to colonize another food source.

What differences are responsible for this remarkable life-history event? Jones et al. (2001) used SAGE (Serial Amplification of Gene Expression) to answer this question; the rest of us are using DNA microarrays (e.g., see http://cmgm.stanford.edu/ kimlab/ wmdirectorybig.html). SAGE is a wonderful but technically tricky method for turning a mixture of cDNAs into a linear polymer of marked 14-mers (tags), each of which usually bears a unique relationship to the transcript of origin. A SAGE library results in a linked series of tags, each of which should be in a one-to-one relationship with an mRNA species. Thus, transcript abundance can be determined by merely sequencing the library and counting the number of occurrences of each SAGE tag. The technical rigor of SAGE, together with the need for large sequencing capacity (the current study used that of the Washington University sequencing facility and involved $\sim 4.5$ million bases of useful sequence), has limited its usefulness. Indeed, in even this amount of sequence, almost $30 \%$ of the tags were seen only once, allowing little statistical power in subsequent analyses. Collectively only $\sim 900$ transcript differences were statistically significant at the $P<.05$ level (we would expect about this many by simple chance)! A second weakness, shared by most transcriptome analyses, is its reliance on genomes of known sequence.

However, SAGE has certain advantages as well. One of these is that SAGE does not depend on the detection of known or even of predicted genes. Instead, SAGE can reveal gene sequences not seen in genome annotations. SAGE can also reveal alternate splicing products that are quite difficult to predict at the genome level. These abilities of SAGE are highlighted in this study. Approximately 33,000 tag species could not be unambiguously assigned for a variety of reasons, and $>12,000$ species had a genomic match but were not in predicted coding regions. One interesting observation that must still be inde- pendently verified is the detection of a high fraction of anti-sense tags from the mitochondrial genome in both the dauer and mixed stage.

A variety of proteins (such as SOD, catalase, HSP90, etc.) have been shown to be stored in dauer larvae at high concentrations, and increased abundance of their transcripts has been reported previously in the dauer or in dauer constitutive mutants (Honda and Honda 1999; Cherkasova et al. 2000). Comparison of these and other candidate genes by Jones et al. revealed the expected differences in most cases. Several antioxidant-protection systems are known to be up-regulated in the dauer larvae, which shows large increases in resistance to oxidants. Antioxidant genes that were up in dauers included sod-3 and sod-4 (but not sod-1 or sod-2), and several of the predicted glutathione peroxidases and catalase $(P=0.10)$.

What unknown products are also packed into the dauer? The most abundant tag (4329 tags in dauer and 215 in mixed-stage) is derived from a genomic region lacking a long open reading frame or protein similarity! It shares properties with known telomerase RNAs and has been named tts-1 (transcribed telomere-like sequence). What is the function of this sequence? Telomerase does not seem to be up in dauers ( 0 vs 6 tags in mixed). There are many other genes up abundantly in dauers and almost lacking in the mixed-stage, or up in mixed-stage and lacking in the dauer. The most prevalent is an $\alpha$ crystallin/ hsp-12.6 homolog that is not induced by heat, although this protein is induced whenever development is arrested by starvation. Evidence for a histone shift, perhaps to stabilize the chromatin, is also evident. Also evident are possible shifts to alternative splice variants in the dauer. As confirmation of these observations, mixed-stage contained many genes obviously involved in nematode growth and development: vitellogenin, actin, and six collagens. Probably the most revealing parts of the study are those unknown genes (15 of the 20 most prevalent) that are up in dauer and not found in mixed-stage and for which a function has still to be ascertained.

For years, it has seemed obvious that the dauer would reveal much about what regu- 
lated the rate of aging. Indeed, by far the best understood set of gerontogene mutants is that which also regulates entrance to the dauer (Braeckman et al. 2001), leading to speculation that the finite life span of $C$. elegans is regulated by a genetic program that evolved to cause lethality (Guarente and Kenyon 2000). What does the Jones paper tell us? First, that the differences between dauer and mixed-stage are incredibly complex, involving as much as $20 \%$ of the entire 19,000 genes of the worm. As is often found in aging analyses, most theories can find support in the data. There is evidence for the oxidative theory with differences in two SOD species and catalase as well as several glutathione reductases being up-regulated in the dauer. There is differential expression of HSPs, especially a not very well-studied one, HSP12, compatible with theories focusing on protein conformation. Then there are also histone transitions, compatible with chromatin remodeling, etc. The outputs of these wholegenome analyses provides grist for the mill of human intelligence in linking such differences to one's favorite model, whatever that is. Better genome annotation and bioinformatics resources are making this enterprise increasingly easy; will we one day be $100 \%$ automated so that all we'll have to do is to purchase the correct software? (But will the software report our results back to its manufacturer surreptitiously in the night?)

An alternate view is that we have now revealed targets and that the next phase is target validation. But what if none of these proteins is key? What if these differences specify an aspect of the dauer other than longevity, or if all of the proteins are needed simultaneously to ensure negligible senescence? Evolutionary biology theory tells us that many changes may be needed (Kirkwood and Austad 2000). Transitions between organisms showing negligible senescence (rockfish, pines) and those undergoing the types of senescence pattern typical of humans and nematodes occur remarkably fast in evolutionary terms (Finch 1990). For humans, such a transition into a negligible mortality state has been the stuff of science fiction for centuries, if not millennia.

In the research lab, transitions in C. elegans mortality rate have been studied for almost two decades, ever since the first convincing demonstration that lifespan (Johnson and Wood 1982; Klass 1983) and mortality rate (Johnson 1987, 1990; Johnson et al. 2001) are under genetic specification. In the last decade, a large number of mutants that specify transitions between the rapid increase in mortality typical of wild type and a lower mortality state have been identified (Braeckman et al. 2001; see http:/ibgwww.colorado. edu/tj-lab/ frame_worm1. html for an up-todate list of all reported gerontogenes). One of the hopes of the new age of genetic studies on aging is that these analyses would reveal general mechanisms underlying the aging process and in particular would reveal the underlying molecular events responsible for these mortality transitions. Interestingly, most gerontogenes implicated by studies in which mutant forms lead to life extension are not themselves differentially up-regulated in the dauer. This includes the first gerontogene age1 , and many others that have been molecularly identified (clk-1, daf-2, gro-1, pdk-1, sir-2, spe-26). Others were up at a variety of statistical significances (akt-1, ins-1 old-1). Two suppressors of gerontogenes were also studied but daf-16 could not be unambiguously detected (another SAGE shortcoming) and daf-18 was found at significantly lower levels (one tag) in the dauer as compared with mixed-stage worms (eight tags).

The DAF-16 protein seems to be at the apex of the regulatory output of the age-1 dauer control pathway (Guarente and Kenyon 2000; Braeckman et al. 2001). DAF16 is a forkhead class transcription factor that is responsible for mediating many of the transitions reported in Jones et al. (2001); the question is how many. Multiple groups are competing to answer this question. One can pull out many probable forkhead binding sites (FK) by surveying the genome upstream of predicted genes but how many of these are real? Not very many, in that only one of the top 25 reported in this paper has a consensus FK sequence upstream. However, it seems clear that DAF-16 responds to both genetic and nongenetic environmental signals and thus integrates multiple inputs, from food availability to temperature, regulating the transition to (and from?) the dauer state. Are there other relevant transcription factors as well? At least one company is betting that human homologs of DAF-16 play a critical role in determining more than human insulin resistance and adult-onset diabetes. Perhaps this target could reveal a molecular switch that allows the in situ transition between senescence (non-negligible mortality) and what most of us want: freedom from disease.

The dauer may be a molecular metaphor for other transitions such as that between worker and queen, regulated by the fabled royal jelly that in nematodes seems to be merely an insulin/IGF-1 ortholog (Guarente and Kenyon 2000; Braeckman et al. 2001). So what have we learned? First, that environmental stress is almost certainly the dominant cause of senescence for nematodes. In the dauer, heat and oxidative stress seem to be guarded against by the up-regulation of a variety of stress response genes. There is no evidence that the longer dauer life is mediated by differential transcription of regulatory gerontogenes. But telomere stabilization and mitochondrial degeneration both have evidence in their favor. Remember, however, that the changes described could be responsible for many other aspects of the dauer morphology and behavior and need not be involved in specifying reduced mortality at all.

Does this lead humans even closer to the fountain, if not of youth, at least of negligible senescence, by following the trail of "Ponce d'elegans", as so aptly quipped by Kenyon (1996)? Evolutionary biologists using theoretical arguments have argued for decades that aging is complex (Rose 1991) but they also predicted that genes such as age-1 that prolong life dramatically could not exist.

Time will tell. If we can live that long!

\section{REFERENCES}

Braeckman, B.P., Houthoofd, K., and Vanfleteren, J.R. 2001. Mech. Aging Devel. 122: 673-693.

Cherkasova, V., Ayyadevara, S., Egilmez, N., and Reis, R.S. 2000. J. Mol. Biol. 300: 433-448.

Finch, C.E. 1990. Senescence, longevity, and the genome. University of Chicago Press, Chicago.

Guarente, L. and Kenyon, C. 2000. Nature 408: 255-262.

Honda, Y. and Honda, S. 1999. FASEB J. 13: 1385-1393.

Johnson, T.E. 1987. Proc. Natl. Acad. Sci. 84: 3777-3781.

-1990. Science 249: 908-912.

Johnson, T.E., Wu, D., Tedesco, P., Dames, S., and Vaupel, J.W. 2001 J. Gerontol. Bio. Sci.(In press)

Johnson, T.E. and Wood, W.B. 1982. Proc. Natl. Acad. Sci. 79: 6603-6607.

Jones, S.J.M., Riddle, D.L., Pouzyrev, A.T., Veculescu, V.E., Hillier, L., Eddy, S.R., Stricklin, S.L., Baillie, D.L., Waterston, R., and Marra, M.A. 2001. Genome Res.11: 1346-1352.

Kenyon, C. 1996. Cell 84: 501-504.

Kirkwood, T.B.L., Braeckman, B.P., Houthoofd, K., and Vanfleteren, J.R. 2001. Mech. Aging Devel. 122: 673-693.

Austad, S.N. 2000. Nature 408: 233-238.

Klass, M.R. 1983. Mech. Aging Dev. 22: 279-286.

Klass, M. and Hirsh, D. 1976. Nature 260: 523-525.

Walker, D.W., McColl, G., Jenkins, N.L., Harris, J., and Lithgow, G.J. 2000. Nature 405: 296-297. 


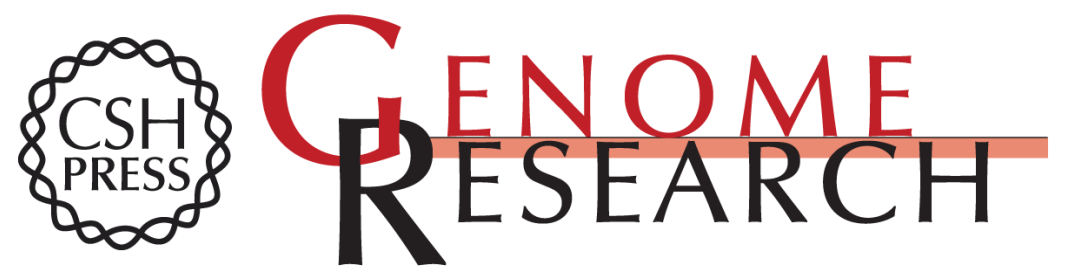

\section{SAGE Thoughts on Aging}

Thomas E. Johnson

Genome Res. 2001 11: 1323-1324

Access the most recent version at doi:10.1101/gr.201101

References This article cites 11 articles, 4 of which can be accessed free at:

http://genome.cshlp.org/content/11/8/1323.full.html\#ref-list-1

\section{License}

Email Alerting Receive free email alerts when new articles cite this article - sign up in the box at the Service top right corner of the article or click here.

\section{Affordable, Accurate Sequencing.}

To subscribe to Genome Research go to: https://genome.cshlp.org/subscriptions 